Chapman University

Chapman University Digital Commons

Pharmacy Faculty Articles and Research

School of Pharmacy

$8-25-2015$

\title{
Rapid Label-free Detection of E. coli using Antimicrobial Peptide Assisted Impedance Spectroscopy
}

Keren Jieng

University of Alberta

Hashem Etayash

University of Alberta

Sarfuddin Azmi

University of Alberta

Selvaraj Naicker

University of Alberta

Mahtab Hassanpourfard

University of Alberta

See next page for additional authors

Follow this and additional works at: https://digitalcommons.chapman.edu/pharmacy_articles

Part of the Bacteria Commons, Environmental Indicators and Impact Assessment Commons, Environmental Monitoring Commons, Fresh Water Studies Commons, Hydrology Commons, Other Environmental Sciences Commons, and the Water Resource Management Commons

\section{Recommended Citation}

Jiang K, Etayash H, Azmi S, et al. Rapid label-free detection of E. coli using antimicrobial peptide assisted impedance spectroscopy. Anal. Methods. 2015;7(23):9744-9748. doi: 10.1039/C5AY01917F

This Article is brought to you for free and open access by the School of Pharmacy at Chapman University Digital Commons. It has been accepted for inclusion in Pharmacy Faculty Articles and Research by an authorized administrator of Chapman University Digital Commons. For more information,

please contact laughtin@chapman.edu. 


\section{Rapid Label-free Detection of E. coli using Antimicrobial Peptide Assisted Impedance Spectroscopy}

\section{Comments}

This is a pre-copy-editing, author-produced PDF of an article accepted for publication in Analytical Methods, volume 7, issue 23 , in 2015 following peer review. The definitive publisher-authenticated version is available online at DOI: 10.1039/C5AY01917F.

\section{Copyright}

Royal Society of Chemistry

\section{Authors}

Keren Jieng, Hashem Etayash, Sarfuddin Azmi, Selvaraj Naicker, Mahtab Hassanpourfard, Parmiss Mojir Shaibani, Garima Thakur, Kamaljit Kaur, and Thomas Thundat 


\section{Analytical Methods}

\section{Accepted Manuscript}

\section{Analytical Methods}
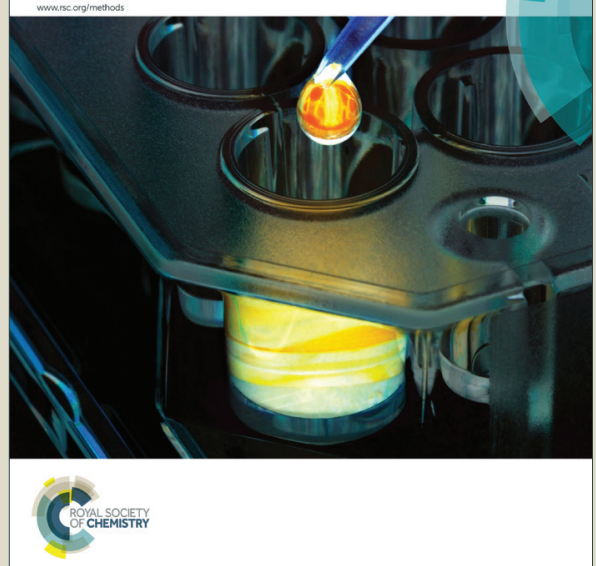

This is an Accepted Manuscript, which has been through the Royal Society of Chemistry peer review process and has been accepted for publication.

Accepted Manuscripts are published online shortly after acceptance, before technical editing, formatting and proof reading. Using this free service, authors can make their results available to the community, in citable form, before we publish the edited article. We will replace this Accepted Manuscript with the edited and formatted Advance Article as soon as it is available.

You can find more information about Accepted Manuscripts in the Information for Authors.

Please note that technical editing may introduce minor changes to the text and/or graphics, which may alter content. The journal's standard Terms \& Conditions and the Ethical guidelines still apply. In no event shall the Royal Society of Chemistry be held responsible for any errors or omissions in this Accepted Manuscript or any consequences arising from the use of any information it contains. 


\section{Analytical Methods}

\section{COMMUNICATION}

\section{Rapid Label-Free Detection of E. coli using Antimicrobial Peptide Assisted Impedance Spectroscopy}

Received xxth XXXX 2015, Accepted xxth XXXX 2015.

DOI: $10.1039 / x 0 \times x 00000 x$
Keren Jiang, ${ }^{a^{*}}$ Hashem Etayash, ${ }^{a, b}$ Sarfuddin Azmi, ${ }^{b}$ Selvaraj Naicker, ${ }^{a}$ Mahtab Hassanpourfard, ${ }^{a}$ Parmiss Mojir Shaibani, ${ }^{a}$ Garima Thakur, ${ }^{a}$ Kamaljit Kaur, ${ }^{b, c}$ and Thomas Thundat ${ }^{{ }^{*}}$

www.rsc.org/

There is an increasing demand for rapid detection of waterborne pathogens to monitor drinking water safety. We demonstrate a compact, label-free sensor array for rapid detection of Escherichia coli (E. coli) in contaminated water samples using antimicrobial peptide assisted impedimetric sensor platform. Interdigitated electrode arrays immobilized with the antimicrobial peptide Colicin V (ColV) were used to screen the affinity towards different bacterial strains by monitoring impedance variations in real-time. This ColV asssisted impedance biosensor exhibited high selectivity towards gram-negative strains particularly towards $E$. coli strains. This selective detection of E.coli from other strains was observed at $10^{2} \mathrm{cfu} \mathrm{mL}^{-1}$, which is clinically relevant. The sensor can detect E. coli from $10^{2}$ to $10^{6} \mathrm{cfu} \mathrm{mL} L^{-1}$ in water sample at $\mathrm{pH} 7$ to 9 . These results show that the antimicrobial peptide ColV assisted impedimetric array is capable of rapid, specific detection of $E$. coli in contaminated water samples.

\section{Introduction}

Escherichia coli (E. coli) is a well-known type of Gram-negative bacteria with certain strains causing serious illness through the synthesis of the Shiga toxin. ${ }^{1}$ The most commonly identified Shiga toxin-producing $E$. coli (STEC) is $E$. coli $0157: \mathrm{H}^{2} .^{2}$ It is estimated that 265,000 STEC infections occur each year in the US. ${ }^{3}$ According to World Health Organization (WHO), E. coli testing is a paramount factor in verifying the microbial safety of water for human consumption. The guideline value of $E$. coli or thermotolerant coliform bacteria in drinking water is $O \mathrm{cfu}$ per $100 \mathrm{~mL}^{4}$ It is becoming increasingly evident that biosensing detection methods should be rapid, sensitive and highly specific in order to avoid further damages caused by life threatening pathogens. The culture and colony counting method may take a long time due to the

\footnotetext{
a. Department of Chemical and Materials Engineering, University of Alberta, Edmonton, Alberta, T6G 2V4, Canada. Emai:Thundat@ualberta.ca; kjiang@ualberta.ca.

${ }^{b .}$ Faculty of Pharmacy and Pharmaceutical Sciences, University of Alberta, Edmonton, Alberta, T6G 2E1, Canada

chapman University School of Pharmacy (CUSP), Harry and Diane Rinker Health Science Campus, Chapman University, Irvine, California, 92618-1908, United States
}

reproduction rate of the bacteria. Other methods including nucleic acid detection (e.g. polymerase chain reaction/PCR), enzyme/substrate method (e.g. bacteria enzymatic activity) and whole-cell recognition (e. g. immunoassay, bacteriophage) are efficient in terms of sensitivity and specificity, but require trained personnel to conduct tests. ${ }^{5,6}$ Currently, most bacteria detection methods are label-dependent, for example, immunoassay, which uses labelled monoclonal antibodies (Mab) to recognize unique bacterial specific antigens. ${ }^{7}$ In addition, most antibodies are expensive and unstable in harsh environment. Thus, there is an urgent need to develop rapid sensors for selective and sensitive detection of $E$. coli in suspected water samples.

In this study, we demonstrate an impedance sensor for rapid detection of $E$. coli in water samples utilizing antibacterial peptide Colicin V (ColV). ColV is an 88 amino acid, linear unmodified antimicrobial peptide (AMP). ${ }^{8}$ It is a type of bacteriocin produced by $E$. coli which acts against $E$. coli strains by binding to the outer membrane receptors and using them to translocate the cytoplasmic membrane, leading to membrane depolarisation, which ultimately kills the cell. ${ }^{8,9}$ In recent years, several studies reported the use of AMPs as pathogen recognition elements in biosensor arrays. They have been proven to be a robust receptor layer with high selectivity towards pathogenic strains of bacteria. ${ }^{10}$ Therefore, we have used ColV as a selective receptor layer for specific recognition of $E$. coli strains in the impedance spectroscopy. By monitoring the impedance amplitude change due to biomolecular binding on the electrodes, impedance spectroscopy offers a rapid, compact, lowcost, and label-free biosensing technique. The sensors have shown enhanced sensitivities and achieved lower detection limits compared to traditional impedance analysers. The impedance amplitude varies with the injected bacteria samples due to the number and type of adsorbed bacteria on the electrode surface. With the ColV functionalized impedance sensor, we have determined the selectivity, detection limit, and the detection capability for $E$. coli contaminated water samples.

\section{Experimental Section}

ColV was genetically expressed and purified from cultures of $E$. coli strain MC4100 as previously described. ${ }^{9}$ E. coli strain ATCC 
25922, L. monocytogenes strain ATCC 19116, S. enteritidis strain ATCC 13076, S. aureus strain ATCC 13566 and P. fluorescens strain $\mathrm{CHAO}$ were used in this study as detection targets.

The impedance sensor array consists of three microfluidic reaction chambers with five pairs of gold interdigitated electrodes (IDEs) in each chamber. The dimensions of each electrode are 3350 $\mu \mathrm{m} / 100 \mu \mathrm{m} / 150 \mathrm{~nm}$ and the spacing between each electrode is 40 $\mu \mathrm{m}$ (Fig 1A and 1B).

The gold electrodes were functionalized with ColV through a covalent bond formation between the free amine group of a thiol linker attached to the electrodes and the carboxylic group on the peptide. The IDEs were functionalized first with a self-assembled monolayer (SAM) of cysteamine linker $\left(\mathrm{HSCH}_{2} \mathrm{CH}_{2} \mathrm{NH}_{2} \cdot \mathrm{HCl}\right)$. The ColV immobilization was performed overnight at room temperature by injection of EDC/NHS activated ColV stock solution. Before each experiment, the functionalized sensor was rinsed with PBS to remove any unbound ColV.

A SHARP IA-2 impedance biosensor (SHARP Laboratory of America, USA) was used to measure the impedance response caused by the affinity binding of the bacteria. When the functionalized ColV sensing layer captures the target bacteria (Fig 1C), the impedance variation caused by the binding of the bacteria is measured in real-time. In order to avoid any interference and eliminate any signal as a result of non-specific adsorption and buffer effects, we used a blank reaction chamber with corresponding buffer/solution as a reference channel for differential readout.

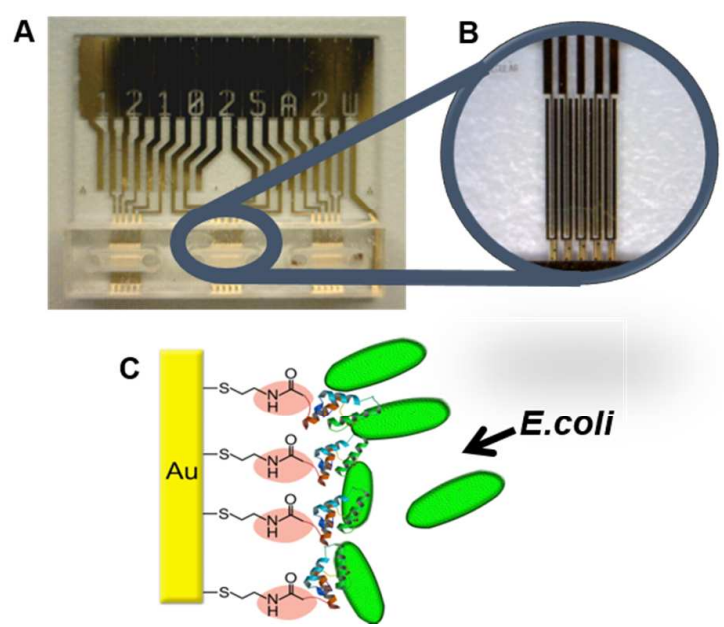

Fig 1. A. Image of the impedance sensor chip; B. Zoomed-in view of the interdigitated electrodes in one microreaction chamber. C. Schematic showing the adsorption of E. coli on the ColV functionalized gold electrode.
The sensitivity, resolution, and the selectivity of the sensor array were tested by injecting $E$. coli and L. monocytogenes with a serial concentration from $10^{2}$ to $10^{6} \mathrm{cfu} \mathrm{mL} L^{-1}$. The bacteria samples were injected into microreaction chambers separately. After the impedance magnitude variation reached an equilibrium state, the bacteria samples, with differing concentration, were injected. To rinse the micro-reaction chambers, PBS was injected into the microreaction chambers after the last sample injection. This also allowed us to test the binding stability between ColV and the bacteria target.

Contaminated artificial beverages and water samples with various pHs were injected into the reaction chambers to test the sensor for unknown solutions using real-world samples. All the test results were monitored and analysed using the Sharp BioZ software package. Impedance magnitude and variations in other parameters were compared to determine the sensor characteristics.

\section{Results and Discussion}

\subsection{Data analysis method}

A Fourier Domain scheme was used for calculating the impedance. The data analysis software package integrated in the Sharp IA-2 user interface was based on a previously described algorithm. ${ }^{11}$ Using surface chemistry theories, the target bacteria bound to the immobilized sensing layer at a rate that follows an exponential function. ${ }^{11,12}$ This rate decays with time after the bacteria is injected into reaction chambers. As a result, the electrode impedance varies as a function of time and it is modelled by:

$$
|Z|=B+A\left(1-e^{-s t}\right)
$$

where $s, A$ and $B$ are constants. $B$ is the offset at which the exponential increase of the impedance modulus begins, and it stands for the impedance baseline of the instrument with the target-free PBS buffer solution. Parameter $s$ expresses the decay time constant which tracks the concentration of the analyte in each reaction chamber. $A$ is related to the sensor surface coverage which relates to the molecular affinity of target-probe interactions. $A$ also represents the "end point" of the binding curve which indicates the density of the sensing layer molecules, size of the analyte particles (bacteria), and dielectric property of the target molecules. From the derivative of the equation (1), the initial slope of the binding curve can be expressed as

$$
\tan \gamma=s \times A
$$

Parameter $s$ can be obtained using the initial slope and the end point of the binding curve. Another parameter of interest is the area covered under the impedance modulus curve which represents the total adsorption amount of the target molecules on the electrode surface. By analysing parameters $A, s$ and area, the probe-target interactions and adsorption kinetics can be studied. ${ }^{12}$ 
A
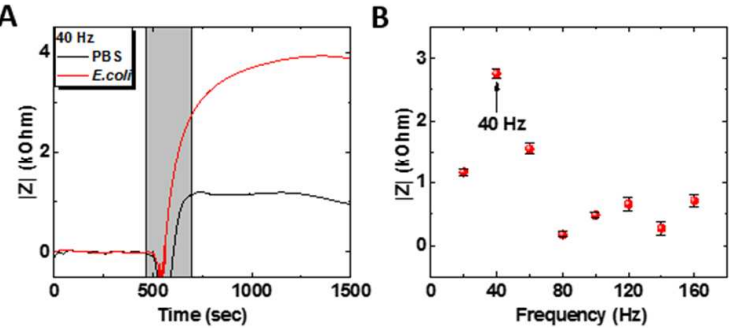

Fig 2. Impedance magnitude increase with the injection of $10^{3}$ cfu $\mathrm{mL}^{-1} E$. coli sample and PBS buffer in the ColV functionalized microreaction chamber. A: impedance magnitude increase at $40 \mathrm{~Hz}$. B: The impedance magnitude differential between E. coli and PBS sample, experiment performed at $40 \mathrm{~Hz}$ gives largest difference between the E. coli sample and PBS buffer.

\subsection{Frequency optimization}

The frequency for optimal operation of the impedance sensor was determined by injecting various samples with and without $E$. coli into the peptide functionalized sensor chambers, and monitoring the response as a function of frequency (20 to $160 \mathrm{~Hz}$ ). Fig $2 \mathrm{~A}$ presents the typical impedance magnitude change with $10^{3}$ cfu $\mathrm{mL}^{-1} E$. coli sample injection. The blank represented the signal of PBS injection in ColV functionalized chamber without target $E$. coli cells. The differential between the E. coli and the blank (net impedance magnitude) was compared at various frequencies (as shown in Fig 2B) and at $40 \mathrm{~Hz}$ the impedimetric magnitude difference was observed to be maximum at $3700 \mathrm{Ohm}$ between the $E$. coli injection and the PBS injection. Thus, $40 \mathrm{~Hz}$ was carefully chosen as the optimum frequency for $E$. coli detection with ColV sensing layer in this study.

\subsection{Selectivity and sensitivity}

The selectivity and sensitivity of this functionalized impedimetric sensor was investigated via net impedance magnitude variation by injecting bacterial solutions at various concentrations (Fig 3A).
From $10^{2}$ to $10^{5} \mathrm{cfu} \mathrm{mL}^{-1}$, the impedance magnitude increases from $1800 \mathrm{Ohm}$ and reaches equilibrium in about $500 \mathrm{sec}$. Response variation for every order of magnitude change in $E$. coli concentration is shown in Fig 3B. After the injection of $10^{6} \mathrm{cfu} \mathrm{mL}^{-1}$ sample of bacteria, the impedance magnitude showed an increase of over $4000 \mathrm{Ohm}$ in about $1000 \mathrm{sec}$. This phenomenon indicates the ColV functionalized electrode surface is fully covered by the bacteria at high concentration and there is physisorption of the bacteria on the electrode surface. However the impedance magnitude variation is minimum with $S$. enteritidis, $S$. aureus, $P$. fluorescens and L. monocytogenes concentration lower than $10^{6} \mathrm{cfu}$ $\mathrm{mL}^{-1}$, indicating that the ColV is less sensitive to other bacteria strains compared to E. coli.

The exponential time constant $s$ is another parameter for the measurement of the AMP-bacteria interaction. The time constant represents the adsorption rate of the bacteria on the electrodes surface. From Fig 3C, the adsorption rate of $E$. coli on the ColV functionalized surface decreases with the bacteria concentration from $10^{2}$ to $10^{5} \mathrm{cfu} \mathrm{mL}^{-1}$. One possible reason is the adsorption of $E$. coli cells reduced the active sites thus the adsorption rate decrease with concentration. However, the time constants of $S$. enteritidis, $S$. aureus, $P$. fluorescens and $L$. monocytogenes injections remained almost unchanged with increasing concentration which shows the ColV sensing layer has weak interaction with other strains.

Mixed samples of $E$. coli and L. monocytogenes were injected into the microreaction chambers in order to test the sensor performance with multiple bacteria strains (purple data curve in Fig 3A, 3B and $\mathbf{3 C}$ ). The impedance magnitude variation and the time constant change of the mixed bacteria injection are similar with that of $E$. coli injections indicating the ColV sensing layer is specific to $E$. coli. For both $E$. coli and the mixed sample, the time constant decrease with the increase of bacteria concentration which suggests the sensor is selective to $E$. coli.

The ColV functionalized impedance sensor showed two orders of magnitude lower detection limit within the short detection time compared with the ELISA method $\left(10^{4} \mathrm{cfu} \mathrm{mL}{ }^{-1}\right) \cdot{ }^{13}$ Also, compared with previous impedimetric bacteria sensors for heat-killed $E$. coli cells, ${ }^{14}$ this Colv functionalized sensor is able to detect live pathogenic $E$. coli cells at a similar concentration range in much shorter detection times. This detection limit is also comparable with impedimetric sensors for Gram-positive bacteria detection

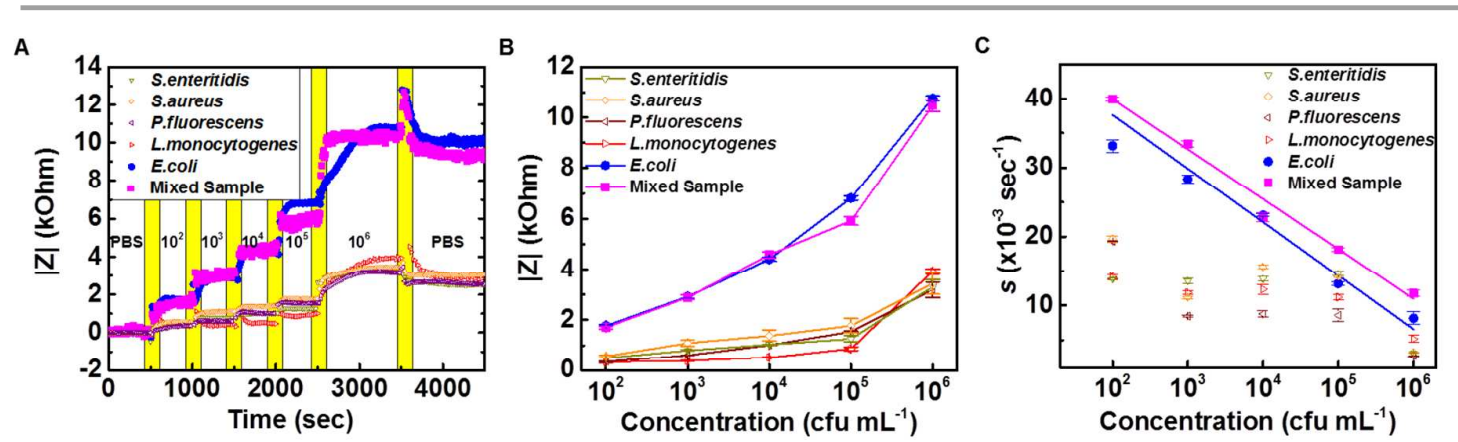

Fig 3. Impedance sensor signal variation via bacteria samples injections. Sensitivity and selectivity of the sensor was compared. A: impedance magnitude change with experiment time; the mixed sample of E. coli and L. monocytogenes represented the similar signal with $E$. coli samples. B: impedance magnitude increase with $E$. coli concentration from $10^{2}$ to $10^{5} \mathrm{cfu} \mathrm{mL}^{-1}$. C: time constant $s$ decrease with $E$. coli concentration, it did not show clear decrease with other strains. 
Table 1. Determination of E. coli concentration in PBS buffer. $E$. coli samples were prepared separately and the impedimetric magnitude change due to $E$. coli samples injection were compared with the previously determined impedimetric magnitude-E. coli concentration relationship in Fig 3B.

\begin{tabular}{cccc}
\hline & $|\mathbf{Z}| \mathbf{( k O h m )}$ & $\begin{array}{c}\text { Measured } \\
\text { Concentration } \\
\left(\mathbf{c f u} \mathbf{~ m L}^{-1}\right)\end{array}$ & $\begin{array}{c}\mathbf{O D}_{600} \\
\text { Concentration } \\
\left(\mathbf{c f u ~ m L}^{-1}\right)\end{array}$ \\
\hline SPL1 & 6.5558 & 110000 & 100000 \\
SPL2 & 5.4815 & 22000 & 20000 \\
SPL3 & 4.3296 & 9900 & 10000 \\
SPL4 & 2.8782 & 1010 & 1200 \\
SPL5 & 3.6715 & 2400 & 2000 \\
\hline
\end{tabular}

developed by Etayash et al. ${ }^{15}$ With this detection method for Gramnegative bacteria, a fast-scan impedimetric sensor with low detection limit for pathogenic bacteria is expected.

\subsection{Artificial sample detection}

E. coli-spiked PBS solutions were prepared as simulated contaminated water samples. The impedance variations due to artificial samples were compared with the impedanceconcentration correlation developed in the sensitivity test (Fig 3B) for estimation of $E$. coli concentration in artificial samples. The $E$. coli concentrations measured using the impedimetric sensor are listed in Table 1.

Comparing with the $\mathrm{OD}_{600}$ concentration, the results measured from the impedance sensor have a $10 \%$ error for concentrations from $10^{4}$ to $10^{5} \mathrm{cfu} \mathrm{mL}^{-1}$. But the error is higher with lower concentration around $10^{3} \mathrm{cfu} \mathrm{mL}^{-1}$, indicating the resolution limit of the sensor. However, from the perspective of bacteria screening, the Colv functionalized impedance sensor is suitable for differentiating pre-concentrated samples at concentrations orders of magnitude greater than the conventional techniques and is accurate within the optimum concentration range.
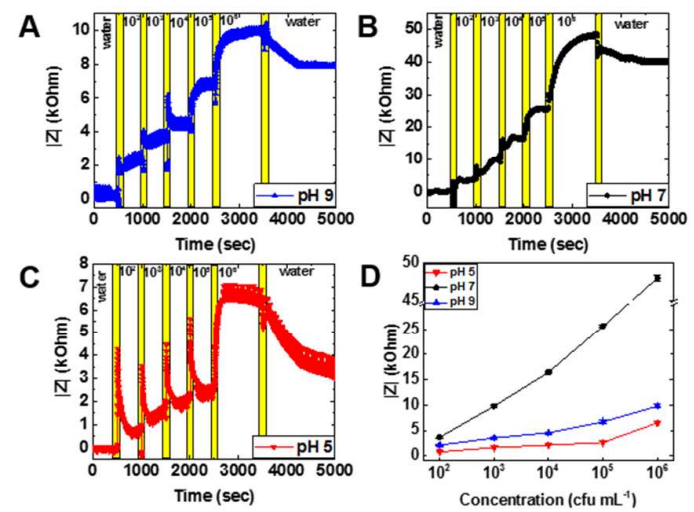

Fig 4. Contaminated water sample detection. E. coli was added into water samples with $\mathrm{pH}$ values of 9,7 and 5 . Impedance magnitude variation with increasing $E$. coli concentration in water at: A. $\mathrm{pH} 9$; B. $\mathrm{pH} 7$; C. $\mathrm{pH}$ 5. D. impedance magnitude increase much higher at $\mathrm{pH} 7$, possibly due to the lower conductivity of $\mathrm{pH} 7$ water. ColV binding activity was inhibited at $\mathrm{pH} 5$.

\subsection{Water sample detection}

Most consumable beverages have a wide $\mathrm{pH}$ range, varying from 3 to 9.5. We prepared water samples with $\mathrm{pH} 5,7$ and 9 by adding $\mathrm{HCl} / \mathrm{NaOH}$ into MilliQ water. Artificially contaminated samples were prepared by adding $E$. coli into the prepared water samples with various concentrations. The contaminated water samples were tested using the Colv functionalized impedance sensor as described in the previous section.

In Fig 4A, 4B and 4C, the impedance magnitudes increase with E. coli concentrations for all three samples. The $\mathrm{pH} 9$ sample represented an identical result as that in PBS buffer which suggests that the sensor is capable of operation at $\mathrm{pH}$ values as high as 9. However, the impedance variation is about five times larger in the $\mathrm{pH} 7$ sample than for the other two (Fig 4D). One possible reason is the lower conductivity at neutral $\mathrm{pH}$. On the other hand, the impedance magnitude increase measured for the $\mathrm{pH} 5$ sample is much lower than previous samples, especially in the concentration range from $10^{2}$ to $10^{5} \mathrm{cfu} \mathrm{mL}^{-1}$. This indicates that ColV binds less at $\mathrm{pH} 5$ than at $\mathrm{pH} 7$ and $\mathrm{pH}$ 9. This dependence of ColV at varying $\mathrm{pH}$ levels was previously reported. ${ }^{16}$ Thus, our ColV functionalized sensor has high sensitivity for $E$. coli detection in water at $\mathrm{pH} 7$ to 9 , but has reduced sensitivity in the lower $\mathrm{pH}$ range.

\section{Conclusion}

Gram-negative antimicrobial peptide ColV has been demonstrated as a bioreceptor in a highly selective and sensitive impedimetric biosensor platform for fast-scan, label-free E. coli detection. Using the impedimetric modulus change, we demonstrated a lower detection limit for E. coli at $10^{2} \mathrm{cfu} \mathrm{mL}^{-1}$ which is clinically relevant. Furthermore, this sensor is able to distinguish $E$. coli from other bacteria strains including $S$. enteritidis, S. aureus, $P$. fluorescens and $L$. monocytogenes. The ColV functionalized sensor was successful in detecting $E$. coli cells in the artificial samples and water samples in the $\mathrm{pH}$ range of 7 to 9 . These results suggest that a ColV assisted impedimetric sensor has the potential to be used in the rapid detection of $E$. coli in water samples with high sensitivity and selectivity. Experiments are presently underway to establish stability and uniformity of this ColV assisted impedance sensor for field applications. . This study also suggests that narrow spectrum antimicrobial peptides can be a good sensing layer for different biosensor platforms such as surface plasmon resonance $(\mathrm{SPR})$ and microcantilevers for label-free detection.

\section{Acknowledgement}

We wish to thank the Natural Sciences and Engineering Research Council of Canada (NSERC) and Canada Excellent Research Chair (CERC) program for supporting this research. We also thank Charles Van Neste for in-depth discussion.

\section{References}

1 M. Varshney, Y. Li, B. Srinivasan and S. Tung, Sens. Actuators B Chem., 2007, 128, 99-107. 
2. M. Barreiros Dos Santos, J.P. Agusil, B. Prieto-Simon, C. Sporer, V. Teixeira and J. Samitier, Biosens. Bioelectron., 2013, 45, 174180.

3 E. Scallan, R. M. Hoekstra, F. J. Angulo, R. V. Tauxe, M. A. Widdowson, S. L. Roy, J. L. Jones and P. M. Griffin, Emerg. Infect. Dis., 2011, 17, 7-15.

4 World Health Organization, Guidel. Drink. Water Qual. Vol. 1 Recomm., 2008.

5 J. D. Slinker, N. B. Muren, A. A. Gorodetsky and J. K. Barton, J. Am. Chem. Soc., 2010, 132, 2769-2774.

6 P. Arora, A. Sindhu, N. Dilbaghi and A. Chaudhury, Biosens. Bioelectron., 2011, 28, 1-12.

7 R. P. Johnson, R. J. Durham, S. T. Johnson, L. A. MacDonald, S. R. Jeffrey and B. T. Butman, Appl. Environ. Microbiol., 1995, 61, 386-388.

8 F. Gerard, N. Pradel and L.F. Wu, J. Bacteriol., 2005, 187, 19451950.

9 V. L. Waters and J. H. Crosa, Microbiol. Rev., 1991, 55, 437-450.

10 M. Varshney and Y. Li, Biosens. Bioelectron., 2007, 22, 24082414.

11 D. S. Messing, A. Ghindilis and K. Schwarzkopf, Conf. Proc. Annu. Int. Conf. IEEE Eng. Med. Biol. Soc. IEEE Eng. Med. Biol. Soc. Conf., 2010, 2010, 2730-2734.

12 A. L. Ghindilis, K. Schwarzkopf, D. Messing, I. Sezan, P. Schuele, C. Zhan, M. Smith, H. Simon and D. Evans, ECS Trans., 2010, 33, 59-68.

13 N. Wang, M. He and H. Shi, Anal. Chim. Acta., 2007, 590, 224231.

14 M. S. Mannoor, S. Zhang, A. J. Link and M. C. McAlpine, Proc. Natl. Acad. Sci. U. S. A., 2010, 107, 19207-19212.

15 H. Etayash, K. Jiang, T. Thundat and K. Kaur, Anal. Chem., 2014, 86, 1693-1700.

16 R. J. Rowbury and M. Goodson, Microbios, 1994, 80, 189-202. 


\section{Rapid Label-Free Detection of $E$. coli using Antimicrobial Peptide Assisted Impedance Spectroscopy}

Keren Jiang, ${ }^{a^{*}}$ Hashem Etayash, ${ }^{a, b}$ Sarfuddin Azmi, ${ }^{b}$ Selvaraj Naicker, ${ }^{a}$ Mahtab Hassanpourfard, ${ }^{a}$ Parmiss Mojir Shaibani, ${ }^{a}$ Garima Thakur, ${ }^{a}$ Kamaljit Kaur, ${ }^{b, c}$ and Thomas Thundat ${ }^{a^{*}}$

a. Department of Chemical and Materials Engineering, University of Alberta, Edmonton, Alberta, T6G 2V4, Canada. Email: kjiang@ualberta.ca; thundat@ualberta.ca.

b. Faculty of Pharmacy and Pharmaceutical Sciences, University of Alberta, Edmonton, Alberta, T6G 2E1, Canada

c. Chapman University School of Pharmacy (CUSP), Harry and Diane Rinker Health Science Campus, Chapman University, Irvine, California, 92618-1908, United States

\section{Graphical Abstract}

A compact, label-free sensor array for rapid detection of Escherichia coli (E. coli) using antimicrobial peptide assisted impedimetric sensor platform.
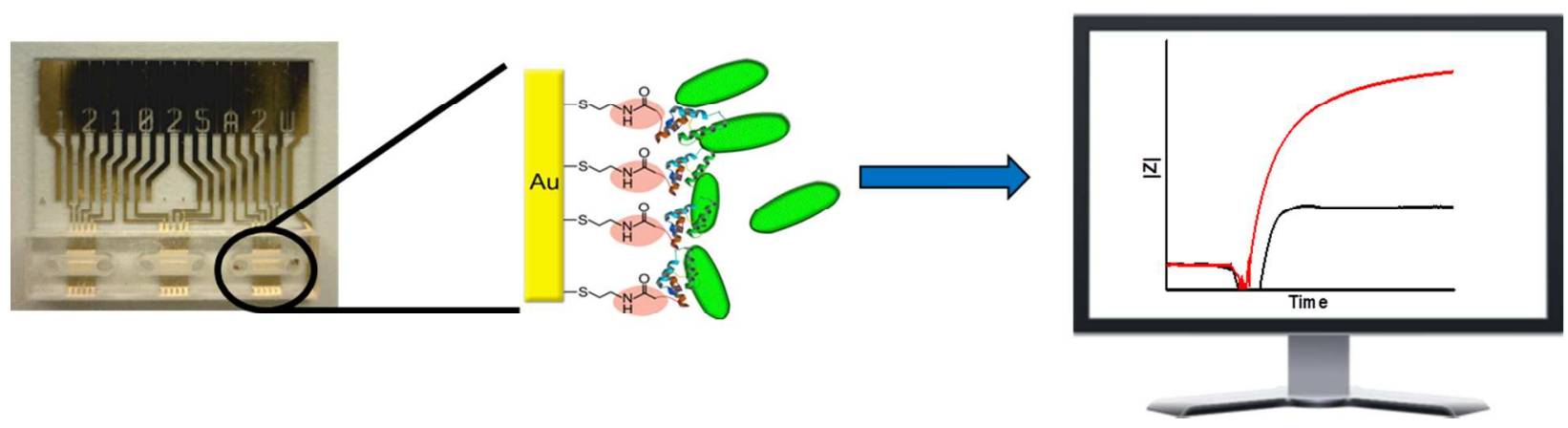